\title{
PERCEPCIÓN DE LOS HABITANTES DE BOGOTÁ SOBRE LA CALIDAD AMBIENTAL, GRADO DE OPTIMISMO Y ATRIBUCIÓN DE RESPONSABILIDAD SOBRE SU DETERIORO ${ }^{1}$
}

\author{
IVÁN FELIPE MEDINA ARBOLEDA* \\ FACULTAD DE PSICOLOGÍA \\ UNIVERSIDAD CATÓLICA DE COLOMBIA \\ PABLO PÁRAMO** \\ UNIVERSIDAD PEDAGÓGICA NACIONAL, COLOMBIA
}

Recibido, noviembre 5/2013
Concepto evaluación, diciembre 10/2013
Aceptado, diciembre 28/2013
Referencia: Medina, I.F. \& Páramo, P. (2014). Percepción de los habitantes de Bogotá sobre la calidad ambiental, grado de optimismo y atribución de responsabilidad sobre su deterioro. Acta Colombiana de Psicología, 17(1), 105-118. doi: 10.14718/ACP.2014.17.1.11

Resumen

\begin{abstract}
Este estudio explora la percepción de habitantes de la ciudad de Bogotá (Colombia) sobre el estado actual del medio ambiente a nivel local, nacional y global, y la prospectiva de cómo estará en el futuro, con el propósito de servir de guía para el desarrollo de los componentes educativos de educación ambiental a la ciudadanía. Igualmente, indaga por la atribución de la responsabilidad del manejo del medio ambiente a futuro. Participaron un total de 118 personas, hombres y mujeres mayores de 18 años, con distintos niveles de formación escolar. El estudio utiliza la Escala de Futuro Ambiental de Gifford et al. (2009) a la que se le introducen dos ítems de relevancia para la evaluación del medio ambiente en Colombia, y la Escala de Atribución de Responsabilidad Ambiental de Barros, Pinheiro y Gunther (2010). Los resultados muestran un pesimismo moderado a nivel nacional, y alto para el caso internacional y local. En cuanto a la atribución de responsabilidad, se identifica un locus de control externo en las causas del deterioro ambiental, siendo los principales agentes mencionados, otras personas y el gobierno nacional. Se discuten los hallazgos en términos de su comparación con estudios de las mismas temáticas en otros lugares del mundo, y sus implicaciones para la promoción del comportamiento proambiental y la atribución de responsabilidad de los bogotanos, así como sobre la contextualización de los medios de comunicación en la valoración del estado del ambiente. Palabras Clave: actitudes ambientales, atribuciones de responsabilidad, escala de futuro ambiental.
\end{abstract}

\section{PERCEPTION OF BOGOTA'S CITIZENS ON ENVIRONMENTAL QUALITY, DEGREE OF OPTIMISM AND ALLOCATION OF RESPONSIBILITY FOR THE IMPAIRMENT}

\begin{abstract}
This study explores the perception of citizens of the city of Bogota (Colombia) on the current state of the environment at local, national and global level, and the prospect of what it will be in the future, in order to provide guidance for the development of environmental components of citizenship education. It also investigates the allocation of responsibility for environmental management in the future. Participants were 118 people, men and women aged over 18 years with different levels of schooling. The instruments used were the Environmental Future Scale by Gifford et al. (2009) to which two items relevant to the assessment of the environment in Colombia were incorporated, and the Allocation Scale for Environmental Responsibility by Barros, Pinheiro and Gunther (2010). Results showed moderate pessimism for the national level, and high for the international and local case. Regarding the allocation of responsibility, an external locus of control was identified in terms of the causes of environmental degradation, where the main actors mentioned were other people and the national government. Findings are discussed in terms of a comparison with similar studies elsewhere in the world and of its implications for promoting proenvironmental behavior and the attribution of responsibility on the part of the citizens of Bogota, as well as the effect of the media assessing the state of the environment.

Key words: environmental attitudes, allocation of responsibility, Environmental Future Scale.
\end{abstract}

\footnotetext{
* Investigador - Asociado, Grupo de Investigación GAEM. Universidad Católica de Colombia. ifmedina@ucatolica.edu.co.

** Docente Profesor Titular, Doctorado Interinstitucional en Educación, Universidad Pedagógica Nacional, Colombia.pdeparamo@gmail.com

1 Los autores expresan sus agradecimientos a los revisores del artículo, sus comentarios fueron fundamentales para el ajuste y revisión de la versión final del artículo.
} 


\title{
PERCEPÇÃO DOS HABITANTES DE BOGOTÁ SOBRE A QUALIDADE AMBIENTAL, GRAU DE OTIMISMO E ATRIBUIÇÄO DE RESPONSABILIDADE AO RESPONSÁVEL DE SEU DETERIORO
}

\begin{abstract}
Resumo
Este estudo explora a percepção de habitantes da cidade de Bogotá, na Colômbia, sobre o estado atual do meio ambiente a nível local, nacional e global, e a prospectiva de como estará no futuro, com o propósito de servir de guia para o desenvolvimento dos componentes educativos de educação ambiental aos cidadãos. Da mesma forma, indaga sobre a atribuição da responsabilidade do manejo do meio ambiente a futuro. Participaram um total de 118 pessoas, homens e mulheres, maiores de 18 anos, com diferentes níveis de formação escolar. O estudo utiliza a Escala de Futuro Ambiental de Gifford et al. (2009) com intens relevantes introduzidos para a avaliação do meio ambiente na Colômbia, e a Escala de Atribuição de Responsabilidade Ambiental de Barros, Pinheiro e Gunther (2010). Os resultados mostram um pessimismo moderado a nível nacional, e alto para o caso internacional e local. Com respeito a atribuição de responsabilidade, identifica-se um locus de controle externo nas causas do deterioro ambiental, sendo os principais agentes mencionados, outras pessoas e o governo nacional. Discutem-se os descobrimentos em termos comparativos com estudos com a mesma temática em outros lugares do mundo, e suas implicações para a promoção do comportamento pró-ambiental e a atribuição de responsabilidade dos bogotanos, bem como sobre a contextualização dos meios de comunicação na avaliação do estado do ambiente.

Palavras chave: atitudes ambientais, atribuições de responsabilidade, escala de futuro ambiental.
\end{abstract}

En los últimos años ha aumentado la preocupación de la sociedad por el medio ambiente, gracias, en parte, a los acuerdos establecidos en la Conferencia de Río de Janeiro de 1992, cuando el gobierno colombiano adquirió compromisos para la protección de los recursos naturales en el mundo, que han venido afinándose en diversas conferencias internacionales (Río + 20, Kyoto, entre otras), y con la creación de instituciones (Ministerio del Ambiente) y de políticas públicas (Política Nacional de Educación Ambiental en 2012) . La difusión de los acuerdos internacionales, así como una posición privilegiada de los temas de protección ambiental en las agendas de los partidos políticos y el trabajo de las ONGs ecologistas, han contribuido a fortalecer los niveles de preocupación sobre el estado del ambiente en el planeta.

En consecuencia, la relación del comportamiento humano con el medio ambiente es un tema de estudio para las diversas disciplinas científicas que dan su aporte a la comprensión de la problemática ambiental y a la búsqueda de las soluciones (para una revisión actualizada del tema véase Guifford, 2014). Particularmente desde las ciencias humanas se ha hecho notar la responsabilidad que tienen los sistemas sociales y culturales sobre una buena parte de los problemas ambientales, al considerar que el comportamiento de las personas en relación con el cuidado del medio ambiente es una de las variables fundamentales para resolver eficientemente los problemas ambientales (Meadows, Meadows \& Randers, 1992; Organización de las Naciones Unidas; 2002).

El énfasis en el comportamiento de los individuos como aspecto clave del cuidado del medio ambiente es retoma- do por la declaración final de la cumbre "Río + 20" en el siguiente apartado:

"Reconocemos que las personas son el elemento central del desarrollo sostenible y, a este respecto, nos esforzamos por lograr un mundo que sea justo, equitativo e inclusivo, y nos comprometemos a trabajar juntos para promover el crecimiento económico sostenido e inclusivo, el desarrollo social y la protección del medio ambiente, lo cual redundará en beneficio de todos"(ONU, 2012, p.2).

El estudio del factor humano, es decir, de la relación entre el comportamiento humano y el impacto en el medio ambiente como aspecto central en la comprensión y solución de las problemáticas ha sido liderado por la psicología ambiental, y se ha centrado en el tema de las actitudes y creencias sobre el medio ambiente, llegando a representar el $25 \%$ del total de publicaciones de las dos revistas más importantes en el área: Environment and Behavior y Journal of Environmental Psychology (Giuliani \& Scopelliti, 2009).

Las actitudes son consideradas como constructos mentales hacia objetos concretos o abstractos (objetos de la actitud), y que pueden ser lugares, cosas, entidades o ideas. Su estudio se considera fundamental porque estarían en la base de la determinación del comportamiento proambiental. (Guifford \& Sussman, 2012).

Las variables que se han estudiado en relación con las actitudes hacia el medio ambiente y su protección han sido la edad, el sexo, la condición social, el lugar de residencia (rural o urbana) y la orientación política de los participantes. 
Así, el estudio de Howell y Laska (1992) reportó que los jóvenes tienen actitudes más favorables hacia el medio ambiente que los adultos. Respecto al sexo, las mujeres muestran una mayor concienciación hacia los problemas ambientales que los hombres (Xiao \& Dunlap, 2007), aunque los resultados no resulten aún concluyentes (Guifford, 2014). En cuanto al nivel educativo, las personas con menores grados educativos y con menores ingresos económicos reportan actitudes menos orientadas hacia la protección del medio ambiente (Shen \& Saijo, 2008), lo cual lleva a considerar que el conocimiento ambiental general es una variable mediadora de las actitudes ambientales (Guifford, 2014).

Por otra parte, los individuos que habitan los grandes centros urbanos muestran, al parecer, mayor preocupación por el deterioro ambiental (Berenguer, Corraliza \& Martín, 2005), y los estudios de los Estados Unidos de América respecto a la ideología política, reportan que quienes se reconocen como liberales muestran una mayor actitud favorable hacia el medio ambiente que los conservadores (Daneshvary et al. 1998).

Con relación a la valoración del estado del ambiente desde el punto de vista espacial o geográfico, los estudios evidencian que por lo general los individuos muestran mayor preocupación por el estado del ambiente a nivel global que en lo local (Dunlap, Gallup \& Gallup, 1993). En la misma dirección está la investigación de Uzzell (2000), en la que también se reporta que los participantes de Australia y algunos países europeos muestran mayor preocupación por los problemas a nivel global que a nivel continental, nacional, local o personal. De aquí que se haya vuelto un eslogan para la educación ambiental que se debe enseñar a "pensar globalmente y actuar localmente", y se plantee la existencia de un sesgo cognitivo en la evaluación del riesgo según la cual, a mayor distancia local y temporal de un evento de análisis, mayor riesgo percibido, dado que el sesgo se reporta en múltiples lugares del mundo (Milfont, Abrahamse \& McCarthy, 2011).

Sin embargo, como lo señalan Hernández, Hidalgo, Salazar-Laplace, y Hess (2010), las conclusiones deben tomarse con precaución por cuanto muchas de las relaciones encontradas entre actitudes favorables hacia el medio ambiente-entendidas como el reporte de los individuos acerca de su comportamiento respecto al cuidado del ambiente, sus sentimientos y creencias sobre el mismo, que típicamente se han medido a través del grado de acuerdo/desacuerdo, o clasificación de importancia, etc. de los individuos frente a un conjunto de afirmaciones-y la presentación de comportamientos responsables con el medio ambiente, son débiles. Así por ejemplo, actitudes ambientales positivas no están necesariamente relacionadas con mejoras prácticas en el uso de pesticidas (Reimer \& Prokopy, 2012); sólo se relacionan con consumo de agua cuando la actitud es medida con mucha especificidad y la actitud es muy positiva (Willis, Stewart, Panuwatwanich, Williams, \& Hollingsworth, 2011). Por esto, la visión de relaciones determinantes actitud -comportamiento ambiental- está dando paso a modelos más comprehensivos que incluyen otros factores, tales como la normatividad local, los incentivos gubernamentales, las normas y prácticas sociales, entre otras (Gadenne, 2011; Papagiannakis, \& Lioukas, 2012).

Por otra parte, se ha reportado que las actitudes pueden estar cambiando con el tiempo, y pueden observarse variaciones importantes de un país a otro. Como se presenta en el trabajo de Gifford et al. (2009), en el que se aplicó la Escala de Futuros Ambientales (EFA) a personas de 18 países del mundo, principalmente de Asia, Europa, y Norteamérica, para evaluar las percepciones de la calidad del ambiente actual y de cómo será ésta en el, futuro en los niveles local, nacional y mundial, se encontró que predomina una visión pesimista del estado del ambiente a futuro, aunque se observaron diferencias entre los países. No obstante, los participantes consideran que el estado del ambiente a nivel local es mejor en sus países que a nivel global (Gifford et al, 2009), resultados que muestran ser consistentes con los informados por Dunlap, el al. (1993) y Uzzell (2000).

El presente trabajo se presenta como una extensión y profundización de la temática abordada en dicho estudio, en la medida en que adopta la EFA para explorar la percepción que tienen los habitantes de Bogotá, D.C. del estado del ambiente, pues no se cuenta con dicha información para ninguna ciudad en Colombia. Identificar estas percepciones resulta importante no sólo porque hasta la fecha no ha habido una aproximación que permita valorar la visión que tienen los bogotanos sobre el estado del medio ambiente a nivel local, nacional y mundial, y su grado de pesimismo/ optimismo frente a estos asuntos, sino porque permite valorar el impacto de las acciones que se han emprendido desde las instituciones gubernamentales y la sociedad civil para promover mayor información y concienciación sobre las problemáticas ambientales y su mitigación en términos de la atribución de responsabilidad que se reporta frente al deterioro o conversación del medio ambiente.

En esa misma dirección, identificar el grado de sensibilidad (pesimismo u optimismo) frente a la problemática ambiental permitirá orientar las nuevas políticas encaminadas a la regulación de la explotación de recursos naturales en el país, siendo ésta una de las políticas de desarrollo establecidas en el Plan Nacional de Desarrollo de los tres últimos periodos presidenciales en Colombia, y una de las fuentes de riqueza nacional (Asociación Colombiana del Petróleo, 2010). Igualmente, evaluar a quién le atribuye 
la población la responsabilidad del estado del ambiente se constituye en un elemento adicional importante para fortalecer o redireccionar los programas educativos ambientales.

Aunque el concepto de atribución se refiere a la valoración que hacen los individuos de sus propios comportamientos (Heider, 1958; Rotter, 1954; Seligman, 1975), bien puede hacerse la extrapolación para indagar si las personas se atribuyen la responsabilidad en el estado futuro del ambiente (atribución interna), o lo hacen a agentes externos (Dios, intereses internacionales, gobierno, suerte, entre otros). Se parte del supuesto de que un individuo orientará sus acciones pro-ambientales dependiendo de si considera que la situación que valora es de su responsabilidad o de otros, así como de la eficacia que se considera puede existir entre el comportamiento y sus efectos sobre el entorno (Sandoval, 2012).

Dado el anterior contexto teórico y empírico, el estudio tuvo como principal propósito investigar las percepciones de los habitantes de Bogotá, D.C., sobre la calidad ambiental a nivel local (ciudad de residencia), regional (país) y global (mundial), así como el grado de optimismo sobre las condiciones futuras del ambiente y la atribución de la responsabilidad de los distintos agentes en el cuidado/ degradación. Por otra parte, se propuso analizar las implicaciones de los resultados para la formulación de políticas ambientales, diseño de programas de educación ambiental $\mathrm{y}$ articulación de relaciones entre actores económicos y sociales para el manejo del tema ambiental.

\section{MÉTODO}

\section{Tipo de estudio}

La investigación es de tipo Descriptivo Correlacional (Montero y León, 2007).

\section{Participantes}

Tomaron parte de este estudio 118 habitantes de Bogotá, D.C. (Colombia), hombres y mujeres de distintos niveles de formación académica. La muestra fue seleccionada a partir de un muestreo no probabilístico, por conveniencia. El criterio de inclusión de la investigación fue: ser mayor de edad y haber vivido más de diez años en la ciudad. En la Tabla 1 se presentan las principales características sociodemográficas de la muestra de participantes.

\section{Instrumentos}

Escala de Futuros Ambientales EFA (Gifford et al. 2009). Se le incorporaron dos nuevos ítems: "manejo de la explotación de las minas" y "manejo de pozos petroleros y oleoductos" por considerar estos aspectos propios de la explotación de los recursos naturales del país. Así, el cuestionario quedó compuesto por 22 ítems que recogen los problemas más comunes del medio ambiente evaluados a partir de dos escalas, una espacial compuesta por tres dimensiones: local, nacional y mundial, y la otra temporal con dos dimensiones: a) condiciones presentes ( muy malo $=1$, a muy bueno=5); y b) condiciones futuras (mucho peor $=-2$ a mucho mejor =2). Se incluyó, además, la opción de respuesta "no sabe" para todas las escalas (véase anexo No.1). La confiabilidad del instrumento original fue de $\alpha=.97$.

La versión del instrumento fue revisada, ajustada en redacción y pertinencia para Colombia por parte de los autores. La versión ajustada fue inicialmente piloteada con 50 participantes, con quienes se evaluó que: a) las instrucciones fueran claras, b) se parafrasearan correctamente las preguntas y c) los ítems se contestaran de acuerdo con las escalas, con el propósito de ajustar la prueba a las características poblacionales colombianas. El puntaje de confiabilidad Alfa de Cronbach de la escala obtenido en el presente estudio es de $=0.94$.

Atribución de responsabilidad (Barros, Pinheiro \& Gunther 2010). Con la cual se indaga sobre el actor (uno solamente) que se considera tendrá más impacto en lo que serán las condiciones ambientales que se presentan en la escala EFA en el futuro.

\section{Procedimiento}

En primer lugar, se localizó a los integrantes de la muestra, se les explicó el propósito del estudio, la forma en que se debían diligenciar los instrumentos, y se solicitaba su consentimiento para que diligenciaran las escalas. El instrumento se aplicó de manera asistida por parte de los investigadores, quienes suministraron las instrucciones y respondieron preguntas que pudieran surgir en el diligenciamiento del mismo.

Tabla 1

Características socio demográficas de la muestra

\begin{tabular}{cccccccc}
\hline $\mathbf{N}$ & Edad & \multicolumn{2}{c}{ Género } & \multicolumn{2}{c}{ Nivel de educación } \\
\hline & Media & Ds & Mujeres & Hombres & Básica & Secundaria & Universitaria \\
\hline 118 & 33 & 13,7 & 55 & 63 & 34 & 67 & 17 \\
\hline
\end{tabular}




\section{RESULTADOS}

Se analizaron 118 escalas. El Alpha de Cronbach para las seis subescalas fue moderado; no obstante, el índice de confiabilidad para la escala total fue de $=0.94$, lo que indica que la versión que se adaptó de la EFA-A posee un alto índice de confiabilidad (ver Tabla 2).

Los resultados de este trabajo se contrastarán posteriormente con los provenientes de otros países de la región: México, Costa Rica, Venezuela, Brasil, Perú, Argentina y Chile.
Tabla 2

Medias y desviación Estándar para cada una de las subescalas de la EFA-A ( $\mathrm{n}=118)$.

\begin{tabular}{lcccc}
\hline & M & DS & A & \% No sabe \\
\hline Local Presente & 2,53 & 0,50 & .64 & 15.00 \\
Nacional Presente & 2,64 & 0,46 & .70 & 12.35 \\
Global Presente & 2,72 & 0,53 & .86 & 22.20 \\
Local Futuro & $-0,26$ & 0,71 & .73 & 16.60 \\
Nacional Futuro & $-0,24$ & 0,75 & .76 & 15.20 \\
Global Futuro & $-0,19$ & 1,09 & .87 & 20.22 \\
\hline
\end{tabular}

Confiabilidad general .943

\section{Dimensión local}

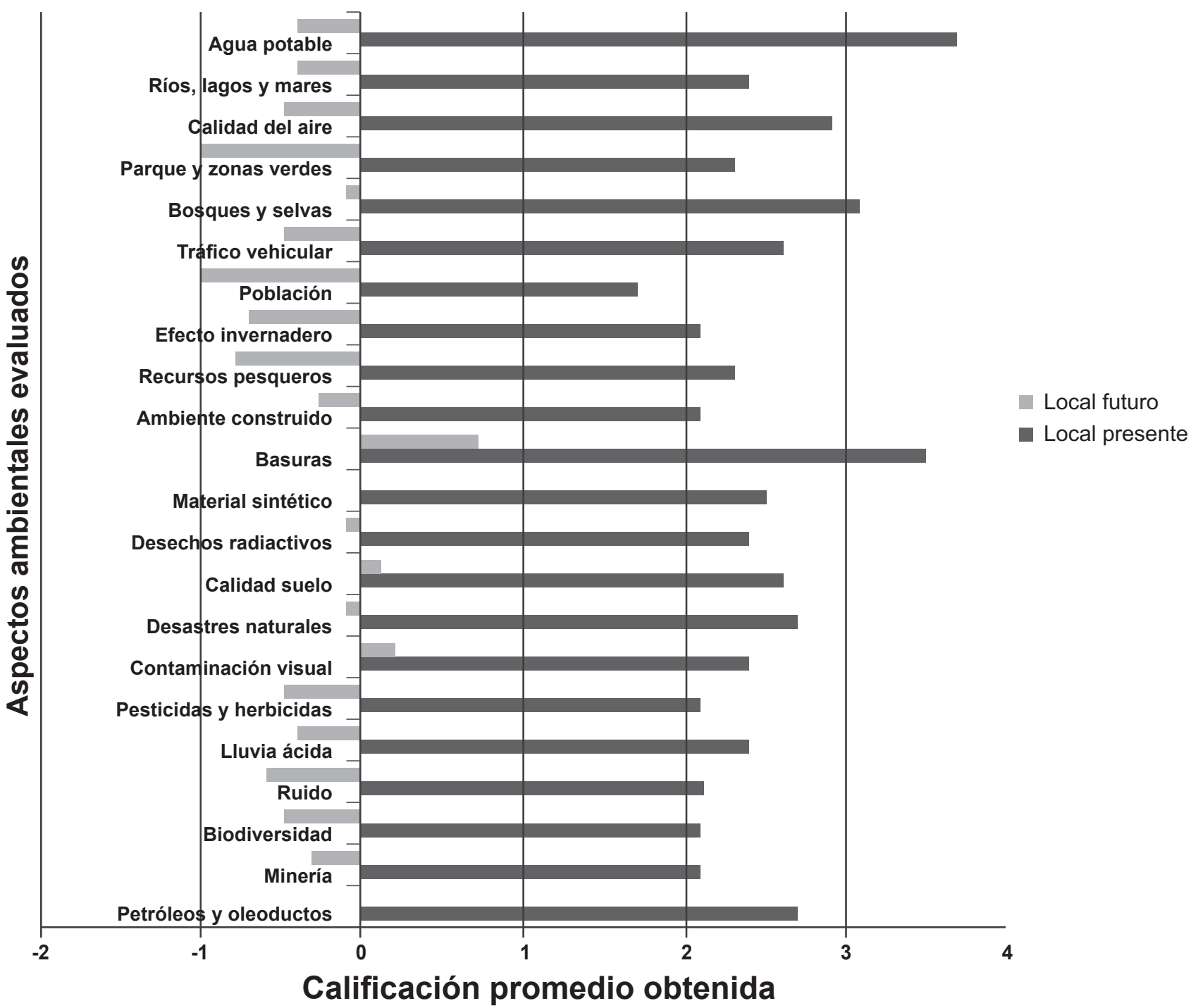

Figura 1. Evaluación media del estado de diferentes aspectos ambientales en el ámbito local en el presente y futuro 


\section{Análisis descriptivo}

A continuación se presentan los resultados para cada una de las dimensiones de las escalas espacial y temporal sobre las que se hizo la indagación;

\section{Evaluación del estado ambiental en el ámbito local}

Presente. La evaluación de las diferentes esferas del ambiente para el caso local (Bogotá, D.C.) en el presente (Ver Figura 1), arroja que los aspectos de Agua Potable y Basuras son evaluados entre aceptable y bueno; la densidad poblacional entre muy malo y malo, mientras que los demás aspectos se consideran entre malos o aceptables. Ningún aspecto ambiental es calificado como bueno o muy bueno, o muy malo.

Futuro. Para la evaluación del futuro, los participantes consideran que estarán igual: el manejo de las basuras, la calidad del suelo y la contaminación visual. Los demás aspectos se evaluaron en general entre igual o peor, siendo los peor evaluados, el control poblacional, los recursos pesqueros y el efecto invernadero. Ningún aspecto fue evaluado mayoritariamente como mucho mejor o mucho peor hacia el futuro.

\section{Dimensión nacional}

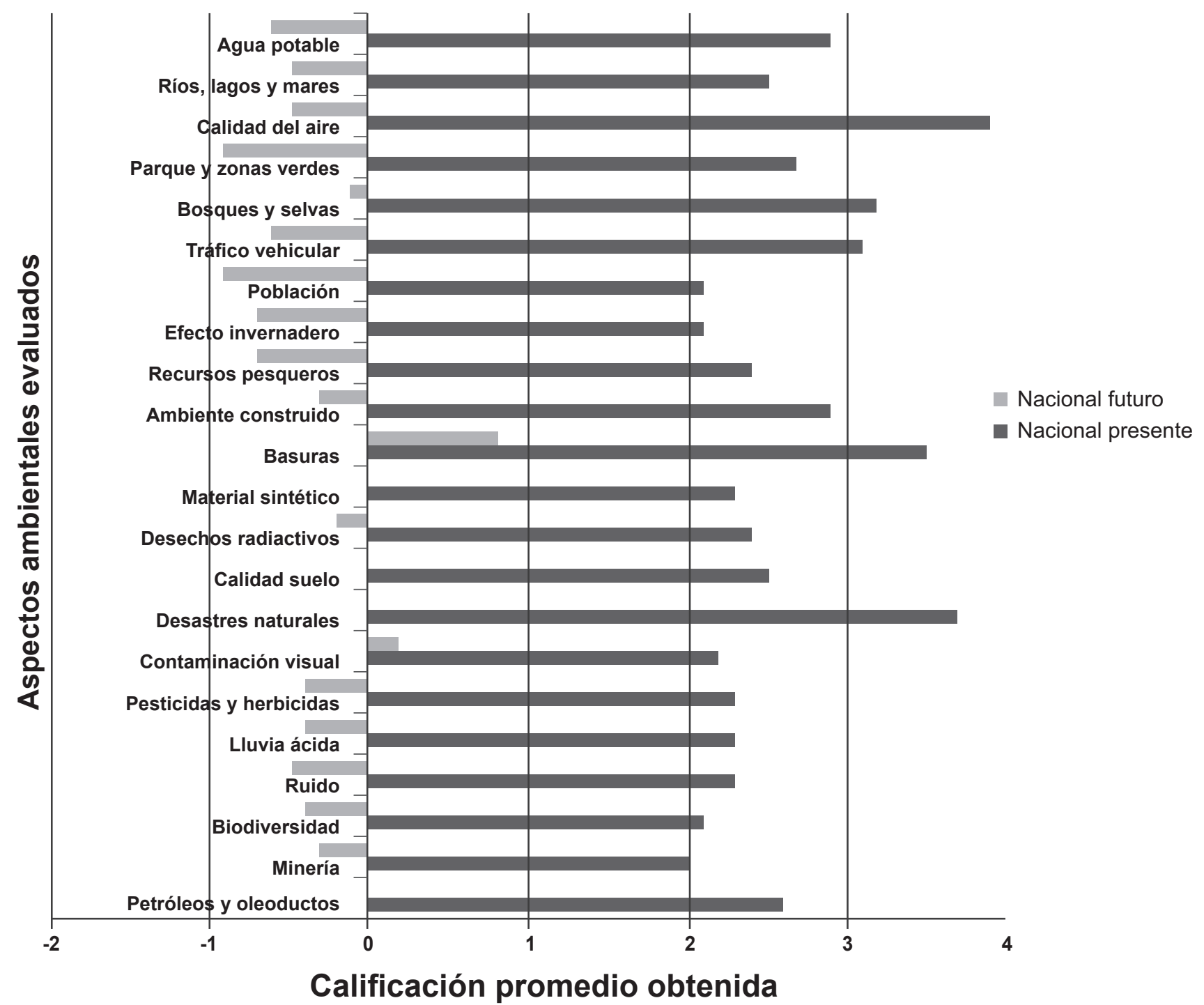

Figura 2. Evaluación media del estado de diferentes aspectos ambientales en el ámbito nacional en el presente y futuro. 


\section{Evaluación del ámbito nacional}

Presente. Para el ámbito nacional, los siguientes aspectos fueron evaluados como aceptables o buenos en el presente: calidad del aire, bosques y selvas, manejo del tráfico, basuras y desastres naturales. Los demás aspectos fueron calificados entre malos y aceptables, siendo particularmente los mayoritariamente calificados como malos: el control poblacional, el efecto invernadero, la contaminación visual, la biodiversidad y la minería (véase Figura 2).

Futuro. Hacia el futuro, se considera que los siguientes aspectos estarán igual o mejor en el país: manejo de basuras y contaminación visual. Los participantes consideran que se mantendrá igual el estado de las selvas y bosques así como el manejo de residuos de fibras y materiales sintéticos, la calidad del suelo y los desastres naturales. Los otros aspectos fueron calificados entre igual o peor, siendo los peor evaluados hacia futuro los parques y zonas verdes y el control de la población.

\section{Evaluación de la dimensión global}

Presente. En la evaluación del estado actual del ambiente en el ámbito mundial se calificaron como aceptables o buenos: la calidad del aire, el estado de bosques y selvas y el manejo de basuras. Los aspectos ambientales que se califican predominantemente como malos son: el control de

\section{Dimensión internacional}

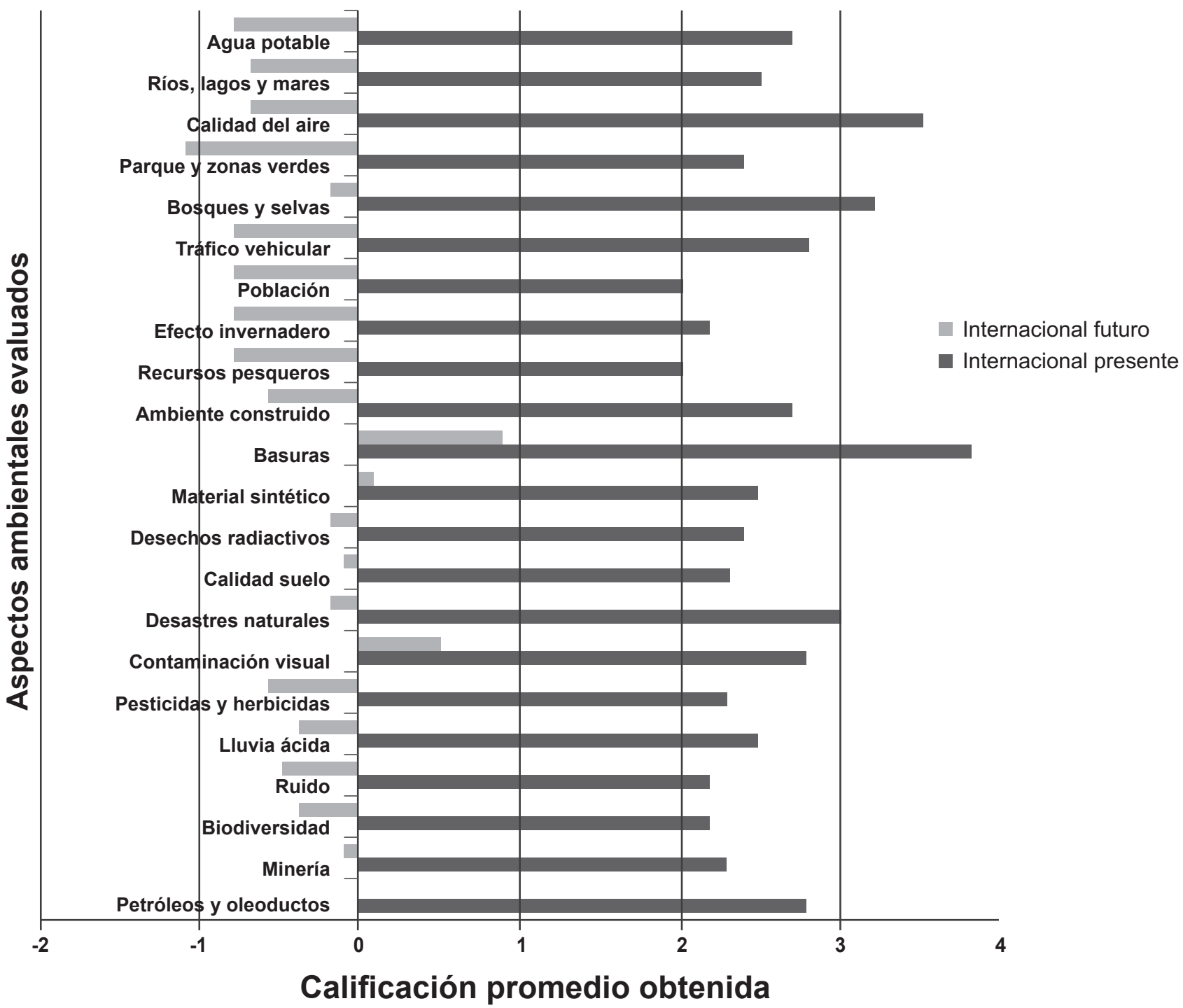

Figura 3. Evaluación media del estado de diferentes aspectos ambientales en el ámbito internacional en el presente y futuro. 


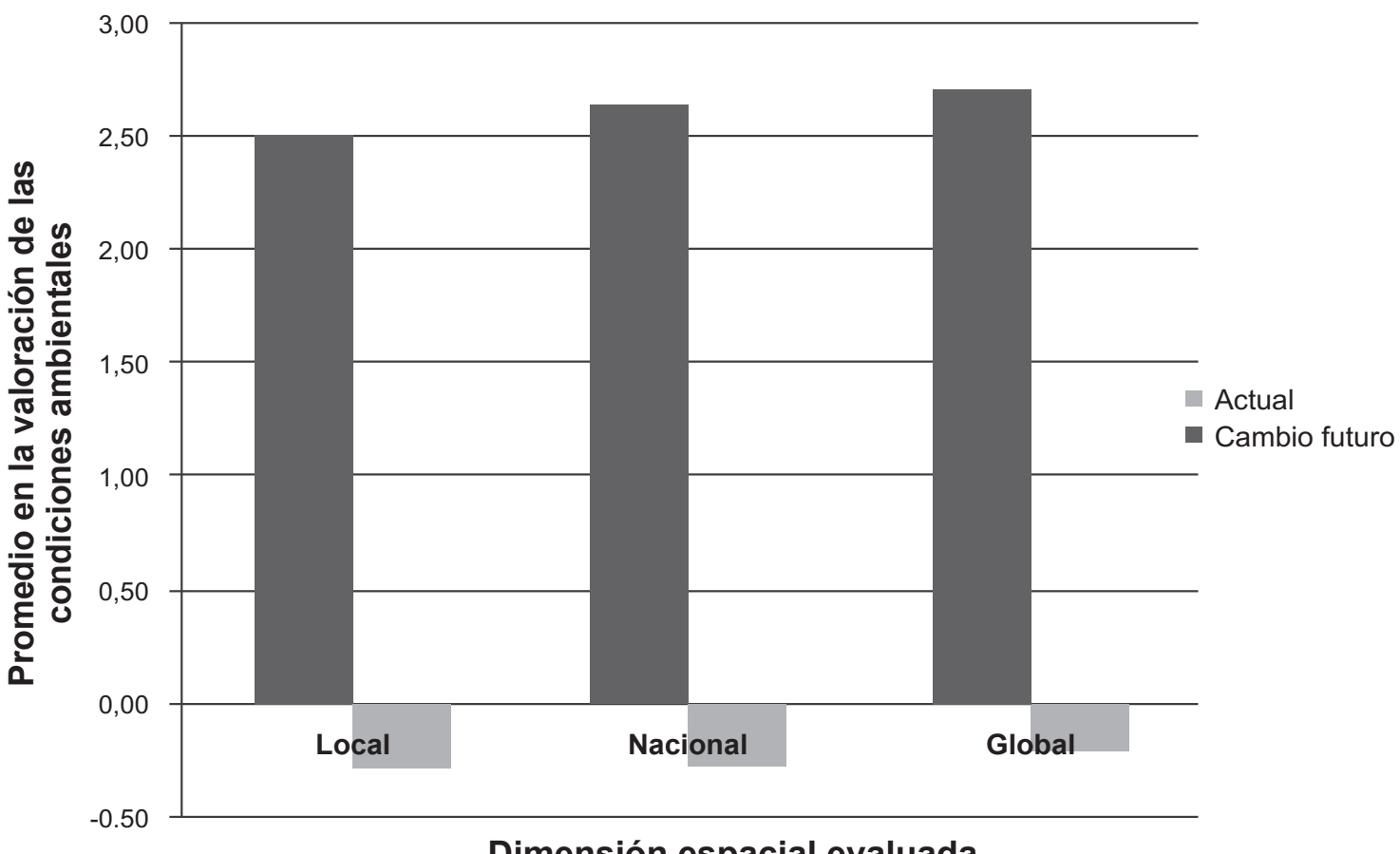

Figura 4. Valoración general de estado del ambiente en las dimensiones Local, Nacional e Internacional.

la población, los recursos pesqueros y el efecto invernadero. Los demás aspectos son calificados en general entre malos y aceptables (véase Figura 3).

Futuro. Hacia el futuro, se considera que estarán peor los siguientes aspectos del ambiente: el acceso al agua potable, los parques y zonas verdes, el tráfico vehicular, el tamaño de la población, el efecto invernadero y los recursos pesqueros. Los siguientes aspectos se considera que estarán mejor o igual: el manejo de las basuras y de la contaminación visual. Se considera que los demás aspectos, en general, seguirán igual o peor.

\section{Evaluación General}

En general los participantes consultados consideran que las condiciones actuales se encuentran entre mal y aceptables para todas las dimensiones espaciales, lo que podría considerarse como un pesimismo moderado, $\mathrm{y}$ hacia el futuro, en general consideran que las condiciones estarán iguales o un poco peor (Véase Figura 4).

Análisis del optimismo espacial en el presente. Para permitir el contraste de los resultados con el estudio de Guilford et al., (2009), se llevó a cabo un análisis estadístico de estas evaluaciones a partir de un ANOVA, al igual que en dicho estudio. El análisis muestra evidencias significativas en las evaluaciones sobre el estado del ambiente para las tres dimensiones espaciales indagadas. En la tabla 3 se presentan los diferentes contrastes estadísticos para la comparación de Optimismo y Dimensión Espacial.

Tabla 3

Contrastes multivariados de Anova de medidas repetidas - Presente.

\begin{tabular}{clccccc}
\hline & \multicolumn{2}{c}{ Contrastes Multivariados } & & \\
\hline Efecto & & Valor & F & Gl de la hipótesis & Gl del error & Sig. \\
\hline GEOGRAFÍA & Traza de Pillai & .132 & $8.850 \mathrm{a}$ & 2.000 & 116.000 & .000 \\
& Lambda de Wilks & .868 & $8.850 \mathrm{a}$ & 2.000 & 116.000 & .000 \\
& Traza de Hotelling & .153 & $8.850 \mathrm{a}$ & 2.000 & 116.000 & .000 \\
& Raíz mayor de Roy & .153 & $8.850 \mathrm{a}$ & 2.000 & 116.000 & .000 \\
\hline
\end{tabular}


Tabla 4

Contrastes multivariados de Anova de medidas repetidas - Futuro

\begin{tabular}{clccccc}
\hline & \multicolumn{7}{c}{ Contrastes Multivariados } \\
\hline Efecto & & Valor & F & Gl de la hipótesis & Gl del error & Sig. \\
\hline GEOGRAFÍA & Traza de Pillai & .025 & $1.468 \mathrm{a}$ & 2.000 & 116.000 & .235 \\
& Lambda de Wilks & .975 & $1.468 \mathrm{a}$ & 2.000 & 116.000 & .235 \\
& Traza de Hotelling & .025 & $1.468 \mathrm{a}$ & 2.000 & 116.000 & .235 \\
& Raíz mayor de Roy & .025 & $1.468 \mathrm{a}$ & 2.000 & 116.000 & .235 \\
\hline
\end{tabular}

En general, las dimensiones local (estado de la ciudad de Bogotá, D.C.) y del mundo son calificadas como peores que el estado del ambiente en el país.

El análisis estadístico de la medida de optimismo para el estado ambiental en el futuro en las tres dimensiones espaciales, local, nacional e internacional, no evidencia diferencias significativas. En la tabla 4 se presentan los diferentes contrastes estadísticos para la comparación de Optimismo sobre el estado del ambiente y Dimensión Espacial.
Para analizar la presencia de optimismo/pesimismo temporal se utilizó la prueba de los signos de Wilcoxon, comparando si el puntaje de cada subescala del futuro (Local futuro, Nacional futuro y Global futuro) diferían significativamente de cero teniendo en cuenta que los puntajes inferiores a cero indicarían pesimismo y los superiores serían un indicio de optimismo sobre el futuro de las condiciones del ambiente (véase Tabla 5).

Este análisis no muestra pesimismo ni optimismo en la medida en que no se observan diferencias significativas

Tabla 5

Prueba de los signos de Wilcoxon comparación de los tres niveles geográficos en el futuro.

\begin{tabular}{lccc}
\hline & $\begin{array}{c}\text { Promedio nacional futuro - } \\
\text { promedio local futuro }\end{array}$ & $\begin{array}{c}\text { Promedio global futuro - promedio } \\
\text { local futuro }\end{array}$ & $\begin{array}{c}\text { Promedio global futuro - promedio } \\
\text { nacional futuro }\end{array}$ \\
\hline $\mathrm{Z}$ & $-1.215^{\mathrm{a}}$ & $-.256^{\mathrm{a}}$ & $-1.204^{\mathrm{b}}$ \\
Sig. asintót. (bilateral) & .224 & .798 & .229 \\
\hline
\end{tabular}

Tabla 6

Índice de correlación de Pearson para variables sociodemográficas y escalas.

\begin{tabular}{lcccccccc}
\hline & & $\begin{array}{c}\text { Nivel } \\
\text { educativo }\end{array}$ & $\begin{array}{c}\text { Local } \\
\text { futuro }\end{array}$ & $\begin{array}{c}\text { Nacional } \\
\text { futuro }\end{array}$ & $\begin{array}{c}\text { Global } \\
\text { futuro }\end{array}$ & $\begin{array}{c}\text { Local } \\
\text { presente }\end{array}$ & $\begin{array}{c}\text { Nacional } \\
\text { presente }\end{array}$ & $\begin{array}{c}\text { Internacional } \\
\text { presente }\end{array}$ \\
\hline Nivel educativo & $\mathrm{P}$ & 1 &,- 244 &,- 251 &,- 205 &,- 039 &,- 051 &,- 042 \\
& $\mathrm{~S}$ & &, 008 &, 006 &, 026 &, 673 &, 586 &, 654 \\
\hline Edad & $\mathrm{P}$ &,- 115 &, 104 &, 132 &, 150 &, 100 &, 090 &, 119 \\
& $\mathrm{~S}$ &, 216 &, 264 &, 153 &, 104 &, 281 &, 334 &, 198 \\
\hline Local futuro & $\mathrm{P}$ &,- 244 & 1 &, 969 &, 866 &, 393 &, 344 &, 421 \\
& $\mathrm{~S}$ &, 008 & &, 000 &, 000 &, 000 &, 000 &, 000 \\
\hline Nacional futuro & $\mathrm{P}$ &,- 251 &, 969 & 1 &, 886 &, 370 &, 374 &, 451 \\
& $\mathrm{~S}$ &, 006 &, 000 & &, 000 &, 000 &, 000 &, 000 \\
\hline Global futuro & $\mathrm{P}$ &,- 205 &, 866 &, 886 & 1 &, 377 &, 380 &, 554 \\
& $\mathrm{~S}$ &, 026 &, 000 &, 000 & &, 000 &, 000 &, 000 \\
\hline Local presente & $\mathrm{P}$ &,- 039 &, 393 &, 370 &, 377 & 1 &, 792 &, 680 \\
& $\mathrm{~S}$ &, 673 &, 000 &, 000 &, 000 & &, 000 &, 000 \\
\hline Nacional presente & $\mathrm{P}$ &,- 051 &, 344 &, 374 &, 380 &, 792 & 1 &, 857 \\
& $\mathrm{~S}$ &, 586 &, 000 &, 000 &, 000 &, 000 & &, 000 \\
\hline Internacional presente & $\mathrm{P}$ &,- 042 &, 421 &, 451 &, 554 &, 680 &, 857 & 1 \\
& $\mathrm{~S}$ &, 654 &, 000 &, 000 &, 000 &, 000 &, 000 & 1 \\
\hline
\end{tabular}

Nota. P. índice de Correlación de Pearson. S. Nivel de significancia. 
$(\mathrm{p} \leq 0,05)$ respecto del valor de cero, por lo cual se afirma que para los participantes colombianos el estado del ambiente en general no variará respecto a las condiciones locales, nacionales o globales en el futuro, aunque la valoración sí muestra un resultado negativo a nivel general sobre el estado futuro del ambiente (véase Tabla 2 y Figura 4).

\section{Análisis de correlaciones}

Para explorar posibles relaciones entre diferentes escalas y las características sociodemográficas de los participantes, se analizó la información mediante correlaciones de Pearson (véase Tabla 6). El nivel educativo fue asumido como variable continua atendiendo a que la escala es sensible a una mayor cantidad de años de permanencia y grados educativos obtenidos en el sistema de educación formal.

En lo que respecta al nivel educativo, no se identifican correlaciones negativas altas; sólo algunas bajas entre el nivel de formación académica de los participantes y la evaluación del estado del ambiente local en el futuro (-,244, p 0,08); el estado del ambiente en el ámbito nacional en el futuro $(-, 251, \mathrm{p} 0,006)$, y el ámbito global en el futuro $(-, 205, \mathrm{p} ., 0,26)$.

Para la variable edad no se identifican relaciones con ninguna de las escalas generales. En lo que respecta a la evaluación del nivel Local Futuro, se encuentra una alta correlación positiva con la forma como se piensa que estará el ambiente nacional en el futuro $(969, \mathrm{p}, 000)$, y la evaluación del ambiente internacional en el futuro (,866, p $, 000)$, lo que indica una alta congruencia en la evaluación de los tres ámbitos hacia el futuro, y correlaciones bajas con los ámbitos en la dimensión temporal presente, siendo la más alta de ellas, con la evaluación de la dimensión internacional presente $(, 421, \mathrm{p}, 000)$.

La evaluación general del estado ambiental Nacional Futuro presenta altas correlaciones positivas con la dimensión

Tabla 7

Porcentaje con que cada uno de los actores fue considerado como el principal responsable del estado del ambiente local en el futuro.

\begin{tabular}{|c|c|c|c|c|c|c|c|c|}
\hline \multirow[t]{2}{*}{ ITEM } & \multicolumn{8}{|c|}{ Agente responsable: $\%$. } \\
\hline & I.I. & G & G.G. & $\mathbf{U}$ & D & $\mathbf{N}$ & $\mathbf{O}$ & N.S. \\
\hline Agua potable & 3,4 & 40,7 & 47,5 & 3,4 & 2,5 & 0 & 0,8 & 1,7 \\
\hline Recurso hídrico & 7,6 & 27,1 & 60,2 & 3,4 & 0,8 & 0 & 0,8 & 0 \\
\hline Biodiversidad & 4,2 & 25,4 & 56,8 & 3,4 & 3,4 & 0,8 & 2,5 & 3,4 \\
\hline Calidad del aire & 4,2 & 22 & 57,6 & 7,6 & 2,5 & 0 & 1,7 & 4,2 \\
\hline Parques y zonas verdes & 2,5 & 34,7 & 55,9 & 5,1 & 0,8 & 0 & 0,8 & 0 \\
\hline Bosques y selvas & 6,8 & 29,9 & 52,1 & 5,1 & 2,6 & 0 & 0,9 & 2,6 \\
\hline Tráfico vehicular & 5,1 & 39,8 & 46,6 & 6,8 & 0 & 0 & 0,8 & 0,8 \\
\hline Población humana & 0 & 11,9 & 72,9 & 5,1 & 1,7 & 0,8 & 3,4 & 4,2 \\
\hline Efecto invernadero & 12 & 17,9 & 49,6 & 3,4 & 8,5 & 0 & 1,7 & 6,8 \\
\hline Recursos pesqueros & 3,4 & 33,6 & 38,8 & 3,4 & 2,6 & 0,9 & 1,7 & 19 \\
\hline Ambiente construido & 3,4 & 37,3 & 45,8 & 3,4 & 0,8 & 0 & 5,1 & 4,2 \\
\hline Basuras & 1,7 & 38,1 & 50,8 & 6,8 & 0,8 & 0,8 & 0,8 & 0 \\
\hline Fibras sintéticas/Materiales sintéticos & 8,5 & 36,4 & 38,1 & 3,4 & 0 & 0,8 & 5,1 & 7,6 \\
\hline Desechos radioactivos & 7,8 & 39,7 & 17,2 & 1,7 & 0,9 & 0,9 & 3,4 & 28 \\
\hline Calidad del suelo & 5,9 & 36,4 & 44,1 & 3,4 & 0 & 0,8 & 3,4 & 5,9 \\
\hline Desastres naturales & 6 & 51,3 & 22,2 & 5,1 & 7,7 & 0,9 & 0 & 6,8 \\
\hline Contaminación visual & 9,3 & 39,8 & 39 & 4,2 & 1,7 & 0 & 3,4 & 2,5 \\
\hline Pesticidas y herbicidas & 5,1 & 35 & 35 & 2,6 & 0,9 & 0,9 & 3,4 & 17 \\
\hline Lluvia ácida & 6,8 & 35,9 & 28,2 & 2,6 & 3,4 & 0 & 5,1 & 18 \\
\hline Ruido & 0,9 & 23,9 & 61,5 & 6,8 & 1,7 & 0 & 0,9 & 4,3 \\
\hline Explotación de minas & 7,6 & 52,5 & 18,6 & 2,5 & 0,8 & 0,8 & 0 & 17 \\
\hline Pozos petroleros y oleoductos & 12 & 50 & 11,9 & 0,8 & 0 & 0 & 0,8 & 25 \\
\hline Promedio & 6 & 35 & 43 & 4 & 2 & 0 & 2 & 8 \\
\hline
\end{tabular}

I.I.: Intereses internacionales, G: Gobierno, 3: Gente en General, 4: Usted, 5: Dios, 6: Nadie, 7: Otros actores, 8: No sabe 
Global Futuro $(, 886, \mathrm{p}, 000)$ y la dimensión Local Futuro $(, 996, p, 000)$. La condición de evaluación del ambiente Local en el presente presenta altas correlaciones positivas con la dimensión nacional presente $(, 792, \mathrm{p}, 000)$ e internacional presente $(.680, \mathrm{p}, 000)$. La dimensión nacional presente también presenta alta correlación positiva con la evaluación internacional presente $(.857, \mathrm{p}, 000)$, identificándose con ello una fuerte correlación entre las dimensiones espaciales para el tiempo presente.

\section{Atribución de responsabilidad}

Dimensión local. Para la evaluación de la atribución de responsabilidad de los agentes que afectan el medio ambiente en la esfera local, los dos agentes que agrupan el mayor porcentaje de responsabilidad según los participantes son: las otras personas y el gobierno, que en promedio agrupan el $78 \%$ de la atribución de responsabilidad. Los agentes que menos afectan el estado del ambiente son: la voluntad de Dios (en promedio 2\%) y la persona que diligencia la encuesta (2\%) (véase Tabla 7).

Dimensión nacional. Para la evaluación de la atribución de responsabilidad de los agentes que afectan el medio ambiente en la esfera nacional (ver Tabla 8), los dos factores que agrupan el mayor porcentaje de responsabilidad de acuerdo con los participantes son las otras personas y el gobierno; en promedio agrupan el $81 \%$ de la atribución de responsabilidad. Los agentes que se considera afectan menos el estado del ambiente como en la dimensión anterior son: la voluntad de Dios (en promedio $2 \%$ ) y la persona que diligencia la encuesta (2\%).

Dimensión global. Para la evaluación de la atribución de responsabilidad de los agentes que afectan el medio ambiente en la esfera global (véase Tabla 9), los dos factores que agrupan el mayor porcentaje de responsabilidad de acuerdo

Tabla 8

Porcentaje en cada uno de los actores fue considerado como el principal responsable del estado del ambiente nacional en el futuro.

\begin{tabular}{|c|c|c|c|c|c|c|c|c|}
\hline \multirow[t]{2}{*}{ ITEM } & \multicolumn{8}{|c|}{ Agente responsable: $\%$. } \\
\hline & I.I. & G & G.G. & $\mathbf{U}$ & D & $\mathbf{N}$ & $\mathbf{O}$ & N.S. \\
\hline Agua potable & 4,2 & 39,8 & 49,2 & 0,8 & 3,4 & 0 & 0,8 & 1,7 \\
\hline Recurso hídrico & 5,9 & 29,7 & 58,5 & 3,4 & 0,8 & 0,8 & 0 & 0,8 \\
\hline Biodiversidad & 7,6 & 30,5 & 49,2 & 2,5 & 2,5 & 0 & 4,2 & 3,4 \\
\hline Calidad del aire & 4,3 & 30,8 & 54,7 & 3,4 & 1,7 & 0 & 2,6 & 2,6 \\
\hline Parques y zonas verdes & 1,7 & 50 & 40,7 & 3,4 & 0 & 0 & 1,7 & 2,5 \\
\hline Bosques y selvas & 6,8 & 36,8 & 50,4 & 1,7 & 2,6 & 0 & 1,7 & 0 \\
\hline Tráfico vehicular & 3,4 & 44,1 & 46,6 & 0,8 & 0,8 & 0 & 0,8 & 3,4 \\
\hline Población humana & 0,8 & 21,2 & 66,9 & 3,4 & 2,5 & 0,8 & 0,8 & 3,4 \\
\hline Efecto invernadero & 15 & 21,2 & 44,1 & 2,5 & 8,5 & 0 & 1,7 & 6,8 \\
\hline Recursos pesqueros & 4,3 & 35 & 43,6 & 3,4 & 1,7 & 0 & 1,7 & 10 \\
\hline Ambiente construido & 2,5 & 44,9 & 42,4 & 0,8 & 2,5 & 0 & 4,2 & 2,5 \\
\hline Basuras & 0,8 & 40,7 & 52,5 & 2,5 & 0,8 & 0,8 & 0,8 & 0,8 \\
\hline Fibras sintéticas/Materiales sintéticos & 7,6 & 39 & 37,3 & 0,8 & 0 & 0,8 & 6,8 & 7,6 \\
\hline Desechos radioactivos & 8,5 & 44,9 & 16,1 & 0,8 & 0,8 & 0,8 & 4,2 & 24 \\
\hline Calidad del suelo & 5,1 & 43,2 & 41,5 & 1,7 & 0 & 0 & 3,4 & 5,1 \\
\hline Desastres naturales & 7,6 & 54,2 & 21,2 & 1,7 & 8,5 & 0,8 & 0 & 5,9 \\
\hline Contaminación visual & 8,5 & 49,2 & 35,6 & 1,7 & 0,8 & 0 & 2,5 & 1,7 \\
\hline Pesticidas y herbicidas & 5,1 & 47,5 & 26,3 & 1,7 & 0 & 0,8 & 5,9 & 13 \\
\hline Lluvia ácida & 6 & 39,3 & 28,2 & 1,7 & 3,4 & 0 & 5,1 & 16 \\
\hline Ruido & 0,9 & 24,8 & 63,2 & 4,3 & 0,9 & 0 & 0,9 & 5,1 \\
\hline Explotación de minas & 11 & 61,9 & 16,9 & 0,8 & 0,8 & 0,8 & 0 & 7,6 \\
\hline Pozos petroleros y oleoductos & 16 & 59,8 & 12 & 0 & 0 & 0 & 0,9 & 11 \\
\hline Promedio & 6 & 40 & 41 & 2 & 2 & 0 & 2 & 6 \\
\hline
\end{tabular}

I.I.: Intereses internacionales, G: Gobierno, 3: Gente en General, 4: Usted, 5: Dios, 6: Nadie, 7: Otros actores, 8: No sabe 
Tabla 9

Porcentaje en cada uno de los actores fue considerado como el principal responsable del estado del ambiente nacional en el futuro.

\begin{tabular}{|c|c|c|c|c|c|c|c|c|}
\hline \multirow[t]{2}{*}{ ITEM } & \multicolumn{8}{|c|}{ Agente responsable: \%. } \\
\hline & I.I. & G & G.G. & $\mathbf{U}$ & D & $\mathbf{N}$ & $\mathbf{O}$ & N.S. \\
\hline Agua potable & 20 & 17,8 & 43,2 & 2,5 & 6,8 & 0 & 1,7 & 7,6 \\
\hline Recurso hídrico & 16 & 17,8 & 49,2 & 2,5 & 5,1 & 0,8 & 0 & 8,5 \\
\hline Biodiversidad & 20 & 17,8 & 43,2 & 5,1 & 5,9 & 1,7 & 6,8 & 0 \\
\hline Calidad del aire & 16 & 12,7 & 57,6 & 3,4 & 4,2 & 0,8 & 0 & 5,1 \\
\hline Parques y zonas verdes & 12 & 30,5 & 42,4 & 4,2 & 3,4 & 0 & 1,7 & 5,9 \\
\hline Bosques y selvas & 19 & 18,8 & 49,6 & 5,1 & 3,4 & 0 & 0,9 & 3,4 \\
\hline Tráfico vehicular & 18 & 26,3 & 45,8 & 0,8 & 0,8 & 0 & 0,8 & 7,6 \\
\hline Población humana & 6,8 & 11,9 & 66,1 & 2,5 & 5,9 & 1,7 & 0 & 5,1 \\
\hline Efecto invernadero & 20 & 17,9 & 40,2 & 1,7 & 10 & 0 & 0,9 & 9,4 \\
\hline Recursos pesqueros & 4,3 & 35 & 43,6 & 3,4 & 1,7 & 0 & 1,7 & 10 \\
\hline Ambiente construido & 7,6 & 31,4 & 47,5 & 1,7 & 2,5 & 0 & 4,2 & 5,1 \\
\hline Basuras & 10 & 28 & 52,5 & 0,8 & 2,5 & 0,8 & 1,7 & 3,4 \\
\hline Fibras sintéticas/Materiales sintéticos & 19 & 30,5 & 30,5 & 0 & 2,5 & 0,8 & 5,1 & 12 \\
\hline Desechos radioactivos & 16 & 39,8 & 15,3 & 0,8 & 4,2 & 0,8 & 4,2 & 19 \\
\hline Calidad del suelo & 12 & 32,2 & 40,7 & 0 & 3,4 & 0 & 2,5 & 9,3 \\
\hline Desastres naturales & 20 & 37,3 & 26,3 & 0,8 & 8,5 & 0,8 & 0 & 6,8 \\
\hline Contaminación visual & 18 & 31,6 & 35,9 & 1,7 & 2,6 & 0 & 5,1 & 5,1 \\
\hline Pesticidas y herbicidas & 15 & 29,1 & 28,2 & 0 & 2,6 & 0,9 & 3,4 & 21 \\
\hline Lluvia ácida & 15 & 28,4 & 29,3 & 3,4 & 5,2 & 0 & 5,2 & 14 \\
\hline Ruido & 11 & 19,5 & 58,5 & 1,7 & 2,5 & 0 & 0,8 & 5,9 \\
\hline Explotación de minas & 25 & 47,5 & 18,6 & 0 & 1,7 & 0 & 0,8 & 6,8 \\
\hline Pozos petroleros y oleoductos & 30 & 48,3 & 11 & 0 & 2,5 & 0 & 0 & 8,5 \\
\hline Promedio & 16 & 28 & 40 & 2 & 4 & 0 & 2 & 8 \\
\hline
\end{tabular}

I.I.: Intereses internacionales, G: Gobierno, 3: Gente en General, 4: Usted, 5: Dios, 6: Nadie, 7: Otros actores, 8: No sabe

con los participantes son igualmente: las otras personas, el gobierno, y los intereses internacionales, agrupando el $81 \%$ de la atribución de responsabilidad. Los agentes que se considera menos afectan el estado del ambiente son de nuevo: la voluntad de Dios (en promedio $2 \%$ ) y la persona que diligencia la encuesta $(2 \%)$.

\section{DISCUSIÓN}

Los resultados de este estudio evidencian que actualmente los participantes tienen, a nivel local, un grado de aceptación moderada frente al estado del ambiente de la ciudad, aunque existe preocupación a futuro por aspectos como el crecimiento poblacional y el efecto invernadero. A diferencia de los hallazgos de otros estudios que evalúan las percepciones del estado del ambiente (Gifford et al, 2009), el nivel nacional y global se ve más positivo que el local, sin que esto signifique que exista una visión optimista en la medida en que los promedios registrados apenas superan el punto medio de la escala en que fue evaluado el estado del ambiente. Por lo general, la percepción es poco optimista, aunque se considera que las condiciones de la ciudad están peores que la situación a nivel nacional. En relación con su visión de futuro, no se registran diferencias respecto al nivel geográfico, local, nacional y global, aunque se mantiene el pesimismo en la medida en que la valoración es negativa a nivel general. El pesimismo frente a las condiciones del ambiente parece ser un visón compartida a nivel mundial si se comparan los hallazgos generales por los informados por Gifford et al (2009). Para el caso colombiano esta situación es comprensible si se considera que el país viene afrontando crisis ambientales durante los periodos invernales de los últimos años, que han repercutido negativamente en los cultivos y han ocasionado derrumbes en carreteras, inundaciones en los hogares, y saldos trágicos en la economía y en vidas 
humanas. Estas situaciones se han difundido por los medios $\mathrm{y}$, en efecto, contribuyen a crear una percepción negativa del estado del ambiente.

Es importante resaltar la atribución de responsabilidad en el estado del ambiente que hacen los encuestados, principalmente, a otras personas, al gobierno y a los intereses internacionales, y la poca responsabilidad que asumen los individuos frente a los problemas ambientales, teniendo en cuenta que si bien no es responsabilidad de un sólo actor, las condiciones actuales del ambiente, el factor del consumo y manejo de los recursos por parte de los individuos, es uno de los aspectos señalados por la Organización de Naciones Unidas como cruciales en la superación de la problemática ambiental global (ONU, 2012).

Por otra parte, hay que rescatar el compromiso de los individuos en el comportamiento de mejora del ambiente cuando se identifica la relación entre el comportamiento y lo que ocurre. En general, el identificar que lo que se hace no está relacionado con modificaciones en el entorno, está asociado con la inacción; este contexto se ha trabajado a profundidad en el marco del comportamiento clínicamente relevante, a propósito de los hallazgos sobre desesperanza adquirida como modelo de la inhibición conductual en los cuadros de depresión (Seligman, 1975). Esta observación puede ser contraproducente si se tiene en cuenta que el impacto de las medidas ambientales pasa por la acción de múltiples individuos, cuyo comportamiento produce resultados que afectan a la población general, en términos de Glenn (1988), como macrocontingencias y metacontingencias.

Los resultados de la investigación son importantes para el país en la medida en que constituyen un indicador de cuánto saben los ciudadanos acerca de la problemática ambiental, y la apreciación del estado de los mismos. Por otra parte, los instrumentos que se usaron para recoger la percepción de los participantes sobre estos aspectos bien podrían servir para evaluar el impacto de los medios, el seguimiento de políticas ambientales y educativas sobre la importancia del uso sostenible de los recursos naturales a nivel local, nacional y global. Así mismo, podrían medir la responsabilidad que tienen los ciudadanos frente a su uso racional, teniendo en cuenta la confiabilidad general de los instrumentos, y su correlación positiva entre dimensiones para las escalas de tiempo presente y futuro.

En ese sentido, es importante destacar cómo la ciudadanía bogotana evalúa como críticos ciertos aspectos de la ciudad en los cuales se sugiere una observancia de los entes de control distrital: tráfico, calidad del aire, sobrepoblación, entre otras. La forma en que se relacionan los individuos con su entorno en términos de su valoración es particularmente importante por el comportamiento de los ciudadanos; la valoración es así vista como una caracteri- zación lingüística de índole implícita que repercute en la ocurrencia de los comportamientos urbanos responsables (Páramo, 2010).

Vale la pena destacar que para futuras caracterizaciones es importante tener en cuenta las diferencias geográficas en la evaluación ambiental del estado del país, pues en este estudio sólo se consideraron las actitudes de participantes de la capital. Con dicho punto de referencia, es importante evaluar en ese mismo sentido las percepciones de ciudadanos de otras ciudades y del área rural para tener un panorama completo de los eventos.

Finalmente, los estudios de percepción pueden complementarse con medidas de comportamiento proambiental distintas al autorreporte, con miras a evaluar la efectividad de medidas tanto en el plano discursivo, en lo que refiere al control verbal sobre el comportamiento humano a través del seguimiento de reglas, como consecuencias sobre el comportamiento, dimensión en la cual finalmente recae la efectividad de los planes de intervención gubernamental para el caso nacional e internacional.

\section{REFERENCIAS}

Barros, H., Pinheiro, J. \& Günther, H. (2010) Who is responsible for the (worsening of) environmental problems? Explorations of Brazilian data. (IAPS 21 Conference, Abstracts of Presentations), June 27- July 2, 2010.

Berenguer, J., Corraliza, J.A \& Martín, R. (2005). Rural-Urban Differences in Environmental Concern, Attitudes and Actions. European Journal of Psychological Assessment, 21, 128-138. http://dx.doi.org/10.1027/1015-5759.21.2.128.

Corral-Verdugo, V., García -Cadena, C. H. \& Frías-Armenta, M. (2010). Psychological approaches to sustainability: Current trends in theory, research and practice. Nova Science Pub Inc.

Daneshvary, N., Daneshvary, R. y Schwe, R.K. (1998). Solidwaste recycling behavior and support for curbside textile recycling. Environmental and Behavior, 30, 144-161. http:// dx.doi.org/10.1177/0013916598302002.

Dunlap, R.E., Gallup, G.H. \& Gallup, A.M. (1993). Of global concern: results of the Health and Planet Survey. Environment, 35, 33-40.

Gadenne, D., Sharma, B., Kerr, D., \& Smith, T. (2011). The influence of consumers' environmental beliefs and attitudes on energy saving behaviours. Energy Policy, 39, 76847694. http://dx.doi.org/10.1016/j.enpol.2011.09.002.

Gifford, R. (2014). Environmental Psychology Matters. Annual review of psychology, 65, 541-579. doi:10.1146/annurevpsych-010213-115048.

Gifford, R. Scannell, L. Kormos, C., Smolova, L., Biel, A., Boncu, S., Corral, V., Güntherf, H., Hanyu, K., Hine, D., 
Kaiser, F., Korpela, K., Lima, L., Mertig, A., García Mira, R., Moser, G., Passafaro, P., Pinheiro, J., Saini, S., Sako, T., Sautkina, E., Savina, Y., Schmuck, P., Schultz, W., Sobeck, K., Sundblad, E. \& Uzzell, D. (2009). Temporal pessimism and spatial optimism in environmental assessments: An 18-nation study. Journal of Environmental Psychology, 29, 1-12. http://dx.doi.org/10.1016/j.jenvp.2008.06.001.

Giuliani, M.V. \& Scopelliti, M. (2009). Empirical research in environmental psychology: Past, present and future. Journal of Environmental Psychology, 29, 375-386. http:// dx.doi.org/10.1016/j.jenvp.2008.11.008

Glenn, S. S. (1988). Contingencies and metacontingencies: Towards a synthesis of behavior analysis and cultural materialism. The Behavior Analyst, 11, 161-179.

Heider, F. (1958). The Psychology of Interpersonal Relations. New York: Wiley.

Hernández, B., Hidalgo, M.C., Salazar-Laplace, M.E. \& Hess, S. (2007). Place attachment and place identity in natives and non-natives. Journal of Environmental Psychology, 27, 310-319. http://dx.doi.org/10.1016/j.jenvp.2007.06.003.

Howell, S.E. \& Laska, S.B. (1992). The changing face of the environmental coalition: A research note. Environmental and Behavior, 24, 134-144. http://dx.doi.org/10.1177/0013916 592241006

Meadows, D.H., Meadow, D.L. y Randers, J. (1992). Más allá de los límites del crecimiento. Madrid: El País Aguilar.

Milfont, T. L., Abrahamse, W., \& McCarthy, N. (2011). Spatial and Temporal Biases in Assessments of Environmental Conditions in New Zealand. New Zealand Journal of Psychology, 40(2), 56-67.

Montero, I., \& León, O. G. (2007). A guide for naming research studies in Psychology. International Journal of Clinical Health \& Psychology, 7(3), 847 - 862.

Organización de las Naciones Unidas (2012) El futuro que queremos. Disponible en: https://rio20.un.org/sites/rio20. un.org/files/a-conf.216-1-1_spanish.pdf.pdf .

Papagiannakis, G. \& Lioukas, S. (2012) Values, attitudes and perceptions of managers as predictors of corporate environmental responsiveness. Journal of Environmental Management, 100, 41-51. http://dx.doi.org/10.1016/j. jenvman.2012.01.023.

Páramo, P. (2010). Aprendizaje situado: creación y modificación de prácticas sociales en el espacio público urbano. Psicologia \& Sociedade, 22(1), 130-138. http://dx.doi. org/10.1590/S0102-71822010000100016.

Reimer, A. \& Prokopy, L. (2012) Environmental attitudes and drift reduction behavior among commercial pesticide applicators in a U.S. agricultural landscape. Journal of Environmental Management, 113(30), 361-369. http://dx.doi. org/10.1016/j.jenvman.2012.09.009.

Rotter (1954). Social Learning and Clinical Psychology. Englewood Cliffs, NJ Prentice - Hall.

Sandoval, M. (2012) Comportamiento sustentable y educación ambiental: una visión desde las prácticas culturales. Revista Latinoamericana de Psicología, 44(1), 181-196.

Seligman, M.E.P. (1975). Helplessness: On Depression, Development, and Death. San Francisco: W.H. Freeman.

Shen, J. \& Saijo,T. (2008). Reexamining the relations between socio-demographic characteristics and individual environmental concern: Evidence from Shangai data. Journal of Environmental Psychology, 28, 42-50. http://dx.doi. org/10.1016/j.jenvp.2007.10.003.

Uzzell, D. L. (2000). The psycho-spatial dimension of global environmental problems. Journal of Environmental Psychology, 20, 307-318. http://dx.doi.org/10.1006/jevp.2000.0175.

Willis, R., Stewart, R., Panuwatwanich, K., Williams, P, \& Hollingsworth, A. (2011). Quantifying the influence of environmental and water conservation attitudes on household and use water consumption. Journal of Environmental Management, 92, 1996 - 2009. http://dx.doi.org/10.1016/j. jenvman.2011.03.023.

Xiao, C \& Dunlap, R.E. ( 2007). Validating a Comprehensive Model of Environmental Concern Cross-Nationally: A U.S. Canadian Comparison. Social Science Quarterly, 88, http:// dx.doi.org/ 10.1111/j.1540-6237.2007.00467.x 471-493. 